\title{
Exosporium gymnemae sp. nov. from India
}

\section{Singh PN*, Baghela A, Singh SK and Maurya DK}

National Fungal Culture Collection of India, Biodiversity and Palaeobiology Group, Agharkar Research Institute, Gopal Ganesh Agarkar Road, Pune, India 411004

Singh PN, Baghela A, Singh SK, Maurya DK 2015 - Exosporium gymnemae sp. nov. from India. Mycosphere 6(5), 508-514, Doi 10.5943/mycosphere/6/5/1

\begin{abstract}
A new species, Exosporium gymnemae is proposed as a saprobe associated with dead stems of Gymnema sylvestre. It has distinct morphological characters, especially the conidiophores which form large, compact, blackish brown synnemata with glazed surface and cylindrical to obclavate conidia with dark bands at the septa. A literature-based checklist of Exosporium species together with their respective hosts and geographic locations is also provided.
\end{abstract}

Keywords - microfungi - Pezizomycotina - taxonomy - Western Ghats.

\section{Introduction}

The Western Ghats comprise mountain ranges in southwestern India that are rich in evergreen rainforests. They are recognized as biodiversity hotspots by virtue of their rich flora and fauna, including endemic species. Our studies on fungal diversity have led to the identification of several novel fungi from this region (Karandikar \& Singh 2010, Rajeshkumar et al. 2012, Senthilarasu \& Singh 2013, Sharma \& Singh 2013, Singh et al. 2009, 2010, 2012). During exploration of fungal diversity in the sacred grooves of Mulshi forest of Western Ghats region of Maharashtra (November 2011), several microfungi were collected for taxonomic study. An interesting effuse, hairy, compact, and glazed fruiting structure around dead stem of Gymnema sylvestre was encountered. This taxon turned out to be a compact synnematous species bearing cylindrical to obclavate conidia with distinct septation pattern and variable in size. Based on its distinct features, the fungus is described as a new species of Exosporium Link.

Exosporium is a hyphomycetous genus with saprobic (Ellis 1961, Zhao 2012) and endophytic or phytopathogenic (Ramakrishnan 1957, Braun \& Nakashima 2014) life styles, and is widely distributed. Kirk et al. (2008) considered there to be about 19 valid species of Exosporium (typified by Exosporium tiliae). Since then, a further five species have been added to the genus, viz., E. livistonae (Crous \& Summerell 2011), E. machili (Zhao \& Zhao 2012), E. husanum (Zhao 2012), E. petersii (Braun 2014) and E. miyakei (Braun \& Nakashima 2014). Table 1 depicts details of validly described species, in the genus.

\section{Materials \& Methods}

\section{Isolates and morphology}

After collection from type locality, specimens were brought to the laboratory in separate paper bags. A trinocular Nikon stereo microscope (Model SMZ-1500 with Digi-CAM, Japan) was 

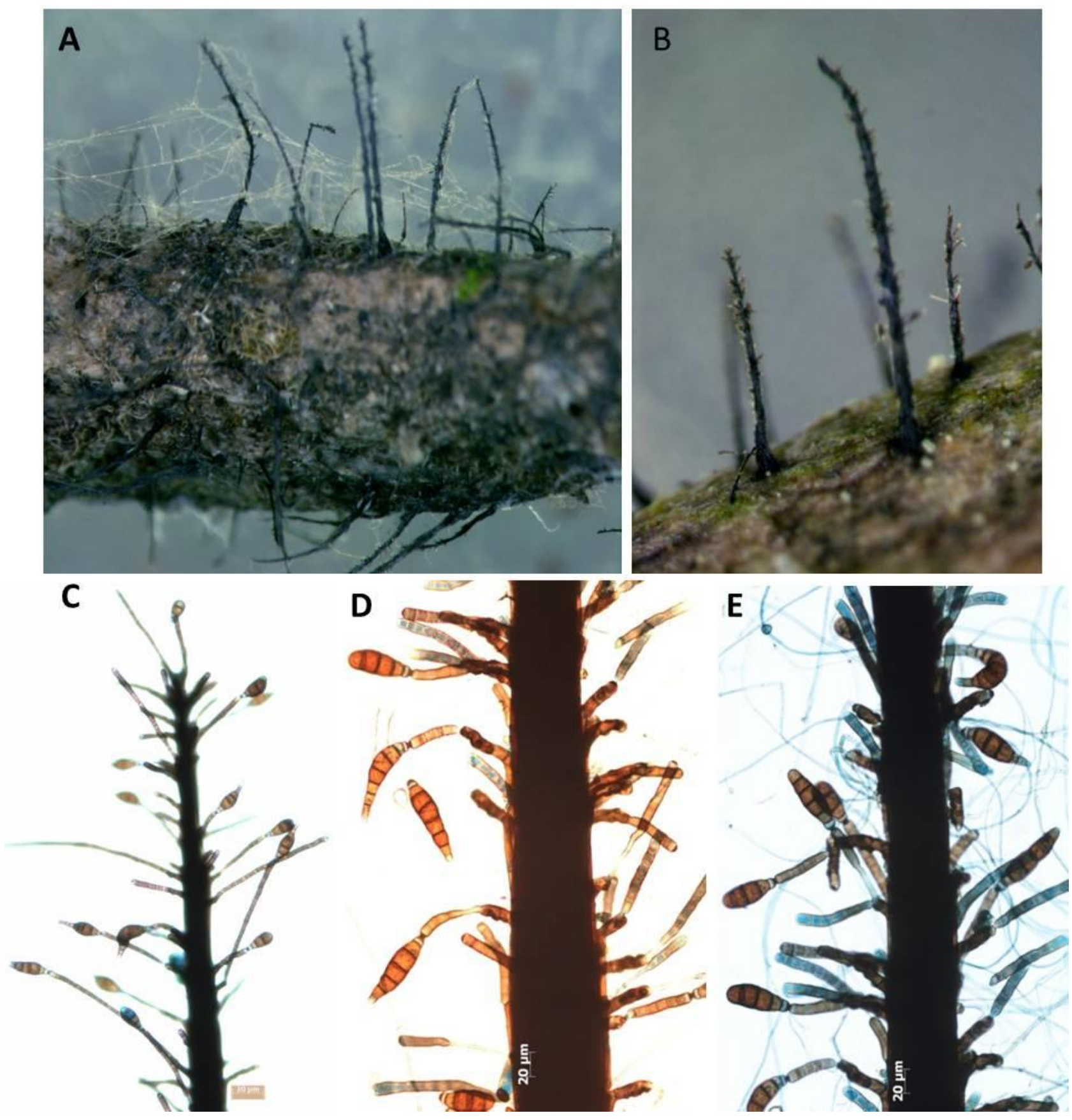

Fig. 1 - Exosporium gymnemae AMH 9666, A: Synnemata on substrate, B: Enlarged view of erect synnmeta arising from substrate (dead stem), C-E: Conidia attached to conidiophores, and dark glazed synnemata. - Bars $C=10 \mu \mathrm{m} ; \mathrm{D}, \mathrm{E}=20 \mu \mathrm{m}$.

used to study features of the fungus on stem surface and for taking photomicrographs. A scrape was taken from growing colonies and mounted in lactic acid cotton blue and examined using an AXIO10 Carl Ziess microscope. Microphotographs were taken of various morphological structures. The holotype specimen is deposited in Ajrekar Mycological Herbarium (AMH), MACS' Agharkar Research Institute, Pune, India with the accession number AMH 9666. The present taxon was compared to the closely related synnematous species of Exosporium.

Attempts to culture the species on artificial media, such as potato dextrose agar and potato carrot agar (Tuite 1969) were unsuccessful. 

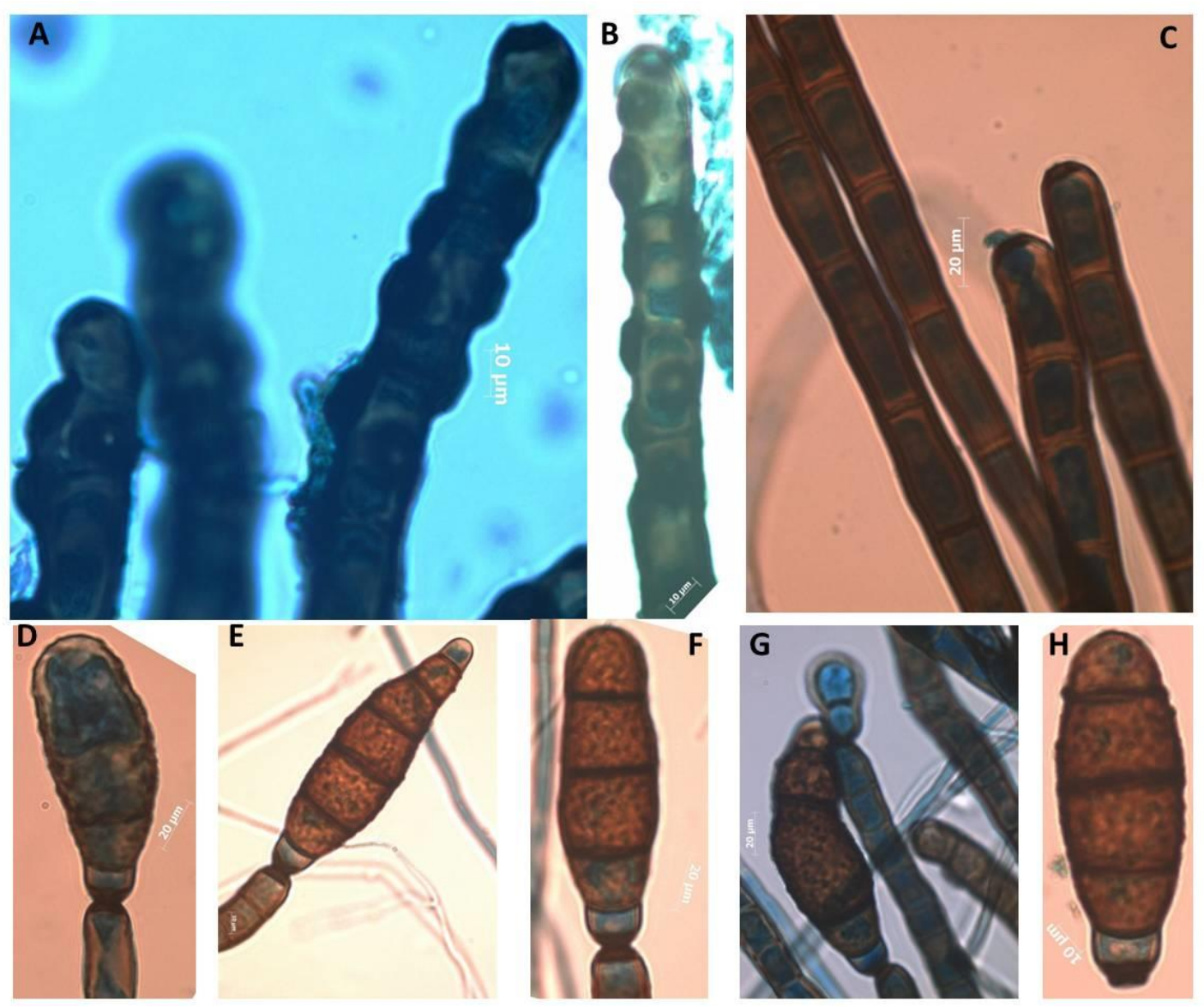

Fig. 2 - Exosporium gymnemae sp. nov. A-B: Polytretic condiogenous cells with prominent circular scars, C: Conidiophores with terminal conidiogenous cells, D-G: Conidia attached to conidiophores, $\mathrm{H}$ : Echinulate conidium with truncate base. - Bars $\mathrm{A}-\mathrm{C}=10 \mu \mathrm{m} ; \mathrm{C}=20 \mu \mathrm{m} ; \mathrm{D}=20$ $\mu \mathrm{m} ; \mathrm{E}=10 \mu \mathrm{m} ; \mathrm{F}, \mathrm{G}=20 \mu \mathrm{m} ; \mathrm{H}=10 \mu \mathrm{m}$.

\section{Results}

\section{Taxonomic description}

Exosporium gymnemae PN Singh, S.K Singh, sp. nov.

Figs $1-2$

MycoBank MB 812842

Type - on dead stem of Gymnema sylvestre R.Br. (Apocynaceae), Sacred Groove (18 $50^{\prime} \mathrm{N}$; $73^{\circ} 50^{\prime}$ E) Mulshi, Distirct Pune, Maharashtra, India, 7 November 2011, coll. P.N. Singh, AMH 9666 (holotype), PNS-ARI 11 (isotype).

Etymology - Latin, gymnemae refers to a generic name of host 'Gymnema'.

Colonies on dead stem punctiform, hairy, brown to black, elongated growing all around the stem surface. Stromata immersed in the substratum, cells compact, pseudoparanchymatous, dark brown. Conidiophores arising from cells of stromata, unbranched, flexuous, septate, dark brown to golden brown, $12.5 \mu \mathrm{m}$ wide at the widest part, closely adpressed forming synnemata, erect, black, glazed surface, wider near the base, 2200-3000 ×50-100 $\mu \mathrm{m}$. Conidiogenous cells integrated, terminal to lateral or intercalary, sympodial proliferation, smooth-walled, olivaceous brown, rarely denticulate, cicatrized, scars prominent (up to 14) forming circular ring, up to $6 \mu \mathrm{m}$ diam., in front view visible as central pore. Conidia acropleurogenous, solitary, straight to slightly curved, clavatocylindric to obclavate, echinulate, thick-walled, pale to dark brown, basal and apical cells sometimes paler or subhyaline, euseptate (2-15 septa), constricted at septa, septation pattern variable (sigmoid, lanceolate and flat band like), base truncate with thick, dark brown scar, apex obtuse to broadly rounded, some conidia with short beak, 21-157 × 16-41.5 $\mu \mathrm{m}$. 


\section{Discussion}

There are four other species of Exosporium that produce synnemata: E. monanthotaxis (Pirozynski 1972), E. ramosum (Ellis 1971), E. assamicum (Agnihothrudu 1966) and E. petersii (Braun 2014). However, E. gymnemae has the largest, glazed, highly compact synnemata, and widest (16-41.5 $\mu \mathrm{m})$, clavato-cylindric to obclavate conidia which are rough walled/echinulate and euseptate. The sigmoid, lanciolate and flat band like septation pattern in new species is also distinct from other species in the genus. Based on these salient features, this taxon is distinct from others in the genus.

Exosporium gymnemae is compared with non-synnematous species of Indian origin. It differs from E. coonoorense (Subramanian 1956) in having shorter and wider conidia. The conidia of E. gymnemae are significantly longer and wider than those of E. bryophylli (Ramakrishnan 1957), E. fici (Payak \& Thirumalachar 1957) and E. ochroleucas (Munjal \& Kulshreshtha 1966). Exosporium lycopersici (Khan \& Sullia 1981), another species described earlier from India, needs to be re-classified as illustrations in the original paper suggest that it is probably a chain forming species of Corynespora (Table 2).

Table 1 Hosts and distribution of Exosporium species.

\begin{tabular}{|c|c|c|c|}
\hline Species & Hosts & Country/Region & References \\
\hline E. acrocomiae & Acrocomia media (leaves) & Puerto Rico & $\begin{array}{lll}\text { Chupp } & \text { ex } & \text { Stevenson } \\
(1975) & & \end{array}$ \\
\hline E. ampullaceum & $\begin{array}{l}\text { Funtumia sp., Rauwolfia sp., } \\
\text { Theobroma sp. (dead twigs and } \\
\text { branches) }\end{array}$ & $\begin{array}{l}\text { Sri Lanka, Ghana, Sierra } \\
\text { Leone, India }\end{array}$ & Ellis (1961) \\
\hline Ex. assamicum & Albizzia chinensis (dead branch) & India (Assam) & Agnihothrudu (1966) \\
\hline Ex. bryophilli & Bryophillum calycinum (leaves) & India (Kerala) & Ramakrishnan (1957) \\
\hline Ex. catenulatum & $\begin{array}{l}\text { Gymnanthes llucida (dead } \\
\text { twigs) }\end{array}$ & Cuba & Ellis (1976) \\
\hline Ex. cinnamomi & $\begin{array}{l}\text { Cinnamomum zeylanicum } \\
\text { (leaves) }\end{array}$ & India (Coorg, Mysore) & Muthappa (1966) \\
\hline Ex. coonoorense & Eugenia sp. (twigs) & India (Coonoor, Madras) & Subramanian (1956) \\
\hline Ex. extensum & Erythrina sp. (dead branch) & Sri Lanka & Ellis (1961) \\
\hline Ex. fici & Ficus benghalensis (leaves) & India (Maharashtra) & $\begin{array}{l}\text { Payak \& Thirumalachar } \\
\text { (1957) }\end{array}$ \\
\hline Ex. husanum & Unidentified (dead twigs) & China (Yunnan) & Zhao (2012) \\
\hline Ex. indicum & Michelia nilgirica (dead twigs) & India (Madras) & Tilak (1966) \\
\hline Ex. lavistonae & Lavistona benthami (leaves) & Australia & $\begin{array}{l}\text { Crous \& Summerell } \\
(2011)\end{array}$ \\
\hline Ex. leptoderridicola & $\begin{array}{l}\text { Leptoderris fasciculata (dead } \\
\text { twigs) }\end{array}$ & Sierra Leone & Ellis (1961) \\
\hline Ex. lycipersici & $\begin{array}{l}\text { Lycopersicon esculentum } \\
\text { (fruits) }\end{array}$ & $\begin{array}{l}\text { India } \quad \text { (Bangalore, } \\
\text { Karnataka) }\end{array}$ & Khan \& Sullia (1981) \\
\hline Ex. machili & Machilus sp. (leaves) & China (Yunnan) & Zhao \& Zhao (2012) \\
\hline Ex. mexicanum & $\begin{array}{l}\text { Erythrina sp., Mascagnia, } \\
\text { Smilax, Uveria (dead stems and } \\
\text { branches) }\end{array}$ & $\begin{array}{l}\text { India, Sri Lanka, Mexico, } \\
\text { New Guinea, Philippines, } \\
\text { Sierra Leone, USA }\end{array}$ & Ellis (1963) \\
\hline Ex. miyakei & Smilax glauca (leaves) & USA (South Carolina) & $\begin{array}{l}\text { Braun \& Nakashima } \\
\text { (2014) }\end{array}$ \\
\hline Ex. monanthotaxis & Monanthotaxis sp. (dead twigs) & $\begin{array}{l}\text { Tanzania, India (Salmalia,), } \\
\text { Puerto Rico, Venezuela }\end{array}$ & Pirozynski (1972) \\
\hline Ex. nattrassii & $\begin{array}{l}\text { Croton macrostachys (dead } \\
\text { branches) }\end{array}$ & Kenya & Ellis (1961) \\
\hline Ex. occidentale & Cornus sp. (dead branches) & $\begin{array}{l}\text { Canada } \\
\text { Saskatchewan) }\end{array}$ & Sutton (1973) \\
\hline Ex. ochroleucum & Dead branch & India (Hamirpur, Punjab) & $\begin{array}{l}\text { Munjal } \\
(1966)\end{array} \quad \& \quad$ Kulshreshta \\
\hline
\end{tabular}




\begin{tabular}{|c|c|c|c|c|}
\hline Species & Hosts & Country/Region & References & \\
\hline Ex. petersii & Smilax nipponica (leaves) & Japan & Braun (2014) & \\
\hline Ex. pterocarpi & Pterocarpus indicus (leaves) & Malaysia & Ellis (1961) & \\
\hline Ex. ramosum & Pinus sp. (dead needles) & USA & Ellis (1976) & \\
\hline Ex. stilbaceum & Elaes sp. (leaves) & $\begin{array}{l}\text { Congo, Ghana,Sudan, } \\
\text { Zambia, Sierra, Leone }\end{array}$ & Ellis (1961) & \\
\hline Ex. tamarindi & Tamarindus indicus (leaves) & $\begin{array}{l}\text { Godavari, Andhra Pradesh, } \\
\text { India }\end{array}$ & $\begin{array}{l}\text { Sydow \& } \\
(1913)\end{array}$ & Sydow \\
\hline Ex. tiliae & Tilia sp. (branches and twigs) & Europe & Link (1809) & \\
\hline E. wisteriae & $\begin{array}{l}\text { Dead branch of Wisteria } \\
\text { sinensis }\end{array}$ & Romania & Sandu (1968) & \\
\hline
\end{tabular}

Table 2 Comparison of morphotaxonomic features of Exosporium gymnemae sp. nov. with synnematous, and non synnematous species of Indian origin.

\begin{tabular}{|c|c|c|c|}
\hline Exosporium spp. & Synnemata & Conidiophores & Conidia \\
\hline E. assamicum & $\begin{array}{l}\text { Up to } 1-\mathrm{mm} \\
\text { long, } 250 \mu \mathrm{m} \\
\text { wide. }\end{array}$ & $\begin{array}{l}\text { Fasciculate, unbranched, slightly darker at } \\
\text { the apex, scars conspicuous. }\end{array}$ & $\begin{array}{l}\text { Cylindrical to obclavate, } 15- \\
\text { distoseptate, } 140-190 \times 24- \\
36 \mu \mathrm{m} \text {. }\end{array}$ \\
\hline E. bryophilli & Absent & $\begin{array}{l}\text { In cluster, erumpent, unbranched, dark } \\
\text { brown, } 140-285 \times 4-6 \mu \mathrm{m} \text {. Scars } \\
\text { conspicuous. }\end{array}$ & $\begin{array}{l}\text { Fusiform or clavate, } 3 \\
\text { euseptate, } 28-25 \times 8-10 \mu \mathrm{m} .\end{array}$ \\
\hline E. coonoorense & Absent & $\begin{array}{l}\text { In cluster, unbranched, darker at base, } \\
\text { paler above, } 770 \mu \mathrm{m} \text { long, } 12 \mu \mathrm{m} \text { thick; } \\
\text { scars thickened and darkend. }\end{array}$ & $\begin{array}{l}\text { Obclavate, } 10-20 \text { euseptate, } \\
90-200 \times 22-33 \mu \mathrm{m} .\end{array}$ \\
\hline E.cinnamomi & Absent & $\begin{array}{l}\text { Fasciculate, unbranched, dark brown, } 200- \\
720 \times 4.5-5.6 \mu \mathrm{m} . \text { Scars thickened. }\end{array}$ & $\begin{array}{l}\text { Obclavate, } 3-\text { distoseptate, } \\
26-60.8 \times 8.5-11 \mu \mathrm{m} .\end{array}$ \\
\hline E. fici & Absent & $\begin{array}{l}\text { Arising in sprodochia, unbranched, } \\
\text { densely compacted, brownish black, } 17- \\
32 \times 5-7 \mu \mathrm{m} .\end{array}$ & $\begin{array}{l}\text { Obclavate to cylindric, } 3-5 \\
\text { euseptate, } 23-92 \times 3.5-7 \\
\mu \mathrm{m} \text {. }\end{array}$ \\
\hline E. indicum & Absent & $\begin{array}{l}\text { Fasciculate, brown to dark brown, } 180- \\
260 \times 9.3-15 \mu \mathrm{m} \text {. Scars thickened. }\end{array}$ & $\begin{array}{l}\text { Obclavate, } 3-8 \text { distoseptate, } \\
90-130 \times 9.3-19 \mu \mathrm{m} .\end{array}$ \\
\hline E. lycopersici & Absent & $\begin{array}{l}\text { Fasciculate, dark brown, thick walled, } \\
218.5-425.5 \times 9-11.5 \mu \mathrm{m} .\end{array}$ & $\begin{array}{l}\text { Obclavate, fusiform to } \\
\text { cylindric, straight or } \\
\text { variously curved, solitary or } \\
\text { catenate, } 2-11 \text { distoseptate, } \\
35-160 \times 7.5-17.5 \mu \mathrm{m} .\end{array}$ \\
\hline E. monanthotaxis & $\begin{array}{l}1-1.5 \mathrm{~mm} \text { long } \\
50-150 \mu \mathrm{m} \text { wide }\end{array}$ & $\begin{array}{l}\text { Conidiophores } 3-4 \mu \mathrm{m} \text { wide near base, } 8- \\
10 \mu \mathrm{m} \text { wide at apex. Scars prominent. }\end{array}$ & $\begin{array}{l}\text { Obovoid to obclavate, } 4-7 \\
\text { distoseptate, } 40-70 \times 15- \\
17 \mu \mathrm{m} \text {. }\end{array}$ \\
\hline E. ochroleucum & Absent & $\begin{array}{l}\text { Fasciculate, unbranched, brown to dark } \\
\text { brown, } 100-380 \times 10-12 \mu \mathrm{m} \text {. Scars } \\
\text { thickened. }\end{array}$ & $\begin{array}{l}\text { Obclavate, } 7-10 \text { distoseptate, } \\
36-100 \times 8-14 \mu \mathrm{m} .\end{array}$ \\
\hline E. petersii & Present & $\begin{array}{l}\text { Loose to dense fasciculate } 2-25 \text { in group, } \\
\text { coremioid, rarely branched, } 30-250 \times 3-6 \\
\mu \mathrm{m} \text {. Conidiogenous cells occasionally } \\
\text { percurrent, enteroblastic. Scars slightly } \\
\text { thickened. }\end{array}$ & $\begin{array}{l}\text { Obclavate, alternarioid, with } \\
\text { very long pale beak in some } \\
\text { conidia, small conidia } \\
\text { broadly fusiform, } 2-11 \\
\text { distoseptate, 20-130 } \times 4-8 \\
\mu \mathrm{m} \text {. }\end{array}$ \\
\hline E. ramosum & Present & $\begin{array}{l}\text { Caespitose, branched towards apex, brown } \\
\text { or dark brown, } 400 \mu \mathrm{m} \text { long, } 6-9 \mu \mathrm{m} \\
\text { wide; scars } 1-4 \text {, conspicuous and dark. }\end{array}$ & $\begin{array}{l}\text { Fusiform to obclavate, } 3-5 \\
\text { euseptate, } 28-38 \times 8-11 \mu \mathrm{m} \text {. }\end{array}$ \\
\hline E. tamarindi & Absent & $\begin{array}{l}\text { Arising in sporodochia, fasciculate, } \\
\text { aseptate, fuliginous, } 4-6 \mu \mathrm{m} \text { wide. }\end{array}$ & $\begin{array}{l}\text { Vermiform, obtuse at the } \\
\text { ends, } 3-10 \text { euseptate, } 18-45 \\
\times 4-6.5 \mu \mathrm{m} \text {. }\end{array}$ \\
\hline E. gymnemae & $\begin{array}{l}2200-3000 \\
50-100 \mu \mathrm{m}\end{array}$ & $\begin{array}{l}\text { Flexuous, septate, dark brown to golden } \\
\text { brown, } 12.5 \mu \mathrm{m} \text { wide at the widest part. }\end{array}$ & $\begin{array}{l}\text { Clavate to obclavate, } 2-15 \\
\text { euseptate, } 21-157 \times 16- \\
41.5 \mu \mathrm{m} .\end{array}$ \\
\hline
\end{tabular}




\section{Acknowledgment}

We are grateful to The Director, MACS-Agharkar Research Institute for providing the necessary facilities to carry out the research.

\section{References}

Agnihothrudu V. 1966 - Notes on fungi from-East India XXI. Two dematiaceous hyphomycetes from India. The Journal of The Indian Botanical Society 44, $398-401$.

Braun U, Crous PW, Nakashima C. 2014 - Cercosporoid fungi (Mycosphaerellaceae) 2. Species on monocots (Acoraceae to Xyridaceae, excluding Poaceae). IMA Fungus 5, 203-390.

Chup C, Stevenson. 1975 - Contrib. Read Herb. 23, 516.

Crous PW, Summerell BA. 2011 - Exosporium livistonae Crous \& Summerell, sp. nov. Persoonia $27,145$.

Ellis MB. 1961 - Dematiaceous Hyphomycetes III. Mycological Papers 82, 1-55.

Ellis MB. 1971 - Dematiaceous Hyphomycetes. Commonwealth Mycological Institute, Kew, UK.

Ellis MB. 1976 - More Dematiaceous Hyphomycetes. Commonwealth Mycological Institute, Kew, UK.

Karandikar KG, Singh SK. 2010 - Lylea indica: a new hyphomycete species from India. Mycotaxon 112, 257-260.

Khan KR, Sullia SB. 1981- A new market disease of tomato caused by Exosporium lycopersici sp. nov. Indian Journal of Mycology and Plant Pathology 11, $122-123$.

Kirk PM, Cannon PF, Minter DW, Stalpers JA. 2008 - Dictionary of the Fungi $10^{\text {th }}$ edition. CAB International, Wallingford, UK.

Link HF. 1809 - Observationes in Ordines Plantarum naturales. Dissertatio I. Magazin Gesellschaft Naturforschender Freunde, Berlin 3, 10.

Munjal RL, Kulshreshtha DD. 1966 - Some dematiaceous hyphomycetes from India IV Exosporium species. Indian Phytopathology 19, 290-293.

Muthappa BN. 1966 - A new species of Exosporium on Cinnamomum zeylanicum L. From India. Sydowia 19, 146-147.

Payak MM, Thirumalachar MJ. 1956 - Notes on some fungi collected on Bombay state (India). Sydowia 10, 30-40.

Pirozynski KA. 1972 - Microfungi of Tanzania. I. Miscellaneous fungi on oil palm. II. New hyphomycetes. Mycological Papers 129, 1-65.

Rajeshkumar KC, Kajale SC, Sutar SA, Singh SK. 2012 - Ellisembia karadkensis sp. nov. from southern Western Ghats, India. Mycotaxon 121, 181-186.

Ramakrishnan T.S. 1957 - Notes on some fungi from South India. VI Proc. Indian Acad. Sci., Pl. Sci. section B 46, 149-154.

Sandu-Ville et al. 1968 - Revue Mycol, (Paris), 33, 78.

Senthilarasu G, Singh SK. 2013 - A new species of Stropharia from Western Ghats, India. Mycotaxon 123, 213-220.

Sharma R, Singh SK. 2013 - A new species of Gymnoascus with verruculose ascospores. IMA Fungus 4, 177-186.

Singh SK, Yadav LS, Singh PN, Hepat R. 2009 - A new species of Gonatophragmium from Western Ghats, India. Mycotaxon 110, 183-187.

Singh SK, Yadav LS, Singh PN, Sharma R, Rajeshkumar KC. 2010 - A new record of Gliocephalotrichum simplex from India. Mycotaxon 114, 163-169.

Singh SK, Yadav LS, Singh PN, Sharma R, Mukherjee G. 2012 - Additions to Gliocephalotrichum species (anamorphic Hypocreales) from fruit litters of the medicinal plants Terminalia chebula in the Western Ghats, India. Mycoscience 53, 391-395.

Subramanian CV. 1956 - Notes on fungi from north east India XXI. Two dematiaceous hyphomycetes from Assam. The Journal of the Indian Botanical Society 35, 53-91. 
Sutton BC. 1973 - Hyphomycetes from Manitoba and Saskatchewan, Canada. Mycological Papers $132,1-143$.

Sydow P, Sydow H. 1913 - Beiträge zur Kenntnis der Pilzflora des Südlichen Ostindiens, I. Annales Mycologici 11, 326-330.

Tilak ST. 1966 - Notes on Indian fungi I. Mycopathologia et Mycologia Applicata 30, 353-356.

Tuite J. 1969 - Plant pathological methods: fungi and bacteria. Burgess Publishing Company, Minneapolis.

Zhao GC. 2012 - Higher microfungi from forests of Yunnan Province Book: 1-572.

Zhao GC, Zhao RL. 2012 - Higher microfungi from forests of Yunnan Province Book: 1-572. 